Écrire

l'histoire

\section{Écrire l'histoire}

Histoire, Littérature, Esthétique

\section{9 | 2019}

\section{L'historien et les langues}

\title{
L'Histoire parle-t-elle?
}

Langage ou mutisme de l'Histoire

\section{Jürgen Trabant}

Traducteur : Philippe Forget

\section{OpenEdition}

\section{Journals}

Édition électronique

URL : http://journals.openedition.org/elh/1997

DOI : 10.4000/elh.1997

ISSN : 2492-7457

Éditeur

CNRS Éditions

Édition imprimée

Date de publication : 1 décembre 2019

Pagination : 171-180

ISBN : 978-2-271-12967-3

ISSN : 1967-7499

Référence électronique

Jürgen Trabant, «L'Histoire parle-t-elle ? », Écrire l'histoire [En ligne], 19 | 2019, mis en ligne le 01 décembre 2019, consulté le 16 février 2021. URL : http://journals.openedition.org/elh/1997; DOI : https://doi.org/10.4000/elh.1997 


\section{L'Histoire parle-t-elle?}

\section{Langage ou mutisme de l'Histoire}

Si par hypothèse, par naïveté ou, pourquoi pas, par métaphore, nous admettons avec Vico que l'Histoire - il s'agit ici de la grande histoire universelle - parle, et si nous nous demandons quel langage elle parle donc aujourd'hui, nous donnerions peut-être une réponse double. Si par langage on entend une langue $e^{*}$ au sens étroit du terme, nous dirons que l'histoire parle anglais. Philologiquement parlant, l'histoire universelle se rapproche cycliquement de son commencement mythique supposé, le paradis, où précisément se parlait une seule langue. Dans ce cas, jamais l'histoire du monde n'a été aussi proche du paradis qu'aujourd'hui. En revanche, si nous entendons par langage un ensemble de signes à travers lequel l'histoire s'adresse à nous, alors il nous faut dire qu'elle parle le langage de la violence. Le paradis ne pourrait pas être plus éloigné. Le mot le plus important de l'histoire la plus récente serait alors de façon certaine cet événement d'une inconcevable brutalité qu'est le 11 Septembre, tout comme ces autres événements d'une inconcevable brutalité - guerre, Auschwitz, guerre - ont été les mots de l'histoire du xx siècle. Ou bien ces événements ne sont-ils en rien les mots d'une histoire qui parle, mais tout au contraire la négation même du langage? Parce que, comme on dit, "quand les armes

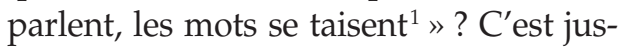
tement pour cette raison que le discours d'Ulrich Beck sur la terreur et la guerre s'intitule Le Silence des mots ${ }^{2}$.

Il n'en reste pas moins que dans les interstices de ces événements brutaux, on parle beaucoup : dans les conférences de la paix, à la Société des Nations, à l'ONU, dans les parlements, etc. En temps de paix, il semble bien que l'histoire parle. Et quand les mots lui manquent, l'histoire paisible émet des signes - celui-ci par exemple, qui est sûrement le mot le plus émouvant et le plus signifiant de notre histoire contemporaine. Ce qui était à dire, l'Allemagne n'a pu le dire autrement qu'à travers le geste qu'illustre ce cliché (voir page suivante).

Mais après? Quand elle a épuisé les ressources de la langue et des signes, l'histoire frappe-t-elle de nouveau avec brutalité, hors langue et signes ? Les mots se taisent-ils alors de nouveau? Est-ce alors l'acte hors langage qui domine?

La langue et les signes ne sont pas aussi innocents qu'ils le paraissent dans cette mise en regard avec la violence, et la violence elle-même n'est pas hors langage. Les mots et les signes ne sont pas seulement absence de violence - ils la rendent aussi possible. En effet, la 


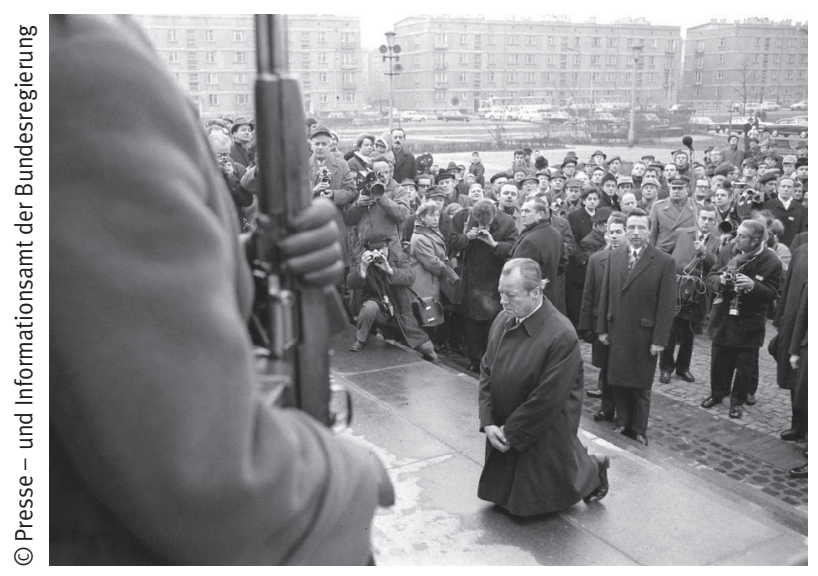

L'agenouillement de Willy Brandt à Varsovie, 7 décembre 1970.

violence dont nous parlons ici n'advient pas hors langage. Dans cet acte de violence qu'est le 11 Septembre, il ne s'agit pas de lions affamés qui se jettent sur la gazelle sans autre forme de procès. Et que ces seigneurs-là se nomment volontiers lions, panthères, loups, etc. (noirs, gris, etc.), ne change rien à l'affaire ; une chaîne infinie d'actes linguistiques et sémiotiques aura été nécessaire pour que le 11 Septembre puisse être organisé - du discours de haine des mollahs jusqu'à l'achat des billets et couteaux et aux ordres donnés aux passagers par les pirates en passant par l'enseignement de l'École technique supérieure de Harburg et les leçons de pilotage -, à travers une langue, des signes, des dessins, des images, des diagrammes. Cet acte - qui dans ce cas précis est en outre éminemment sémiotique dans la mesure où il est aussi voulu comme signe - est pris dans des actes linguistiques et sémiotiques qui font son lit. La même chose vaut pour la guerre, les camps de concentration et les exils forcés : tout cela est inséparable du fait que les hommes sont des êtres de parole qui produisent des signes. Sans langage, tout cela n'existerait pas.
Depuis la Chute, c'est-à-dire depuis le début, langue et signes sont partout où agit l'humain.

De fait, l'histoire parle.

Cette prise de conscience est au fondement du premier - et dans une certaine mesure inoffensif - linguistic turn ${ }^{3}$ de la science historique actuelle. C'est lui qui a fait que les mots de l'histoire - ceux par lesquels s'exprime l'événement, les signes qui sont l'événement même, les événements qui sont signes, et le tressage communicationnel dans lequel l'action est prise - sont devenus l'objet de l'observation historique. Le caractère langagier de l'histoire s'impose avec une intensité variable à la conscience des historiens, et il occupe dans leurs recherches un espace d'ampleur variable: chez Koselleck par exemple, qui opère déjà un véritable linguistic turn, l'histoire des concepts accompagne et complète l'histoire sociale. Chez Chartier ${ }^{4}$, Darnton ${ }^{5}$ ou Davis ${ }^{6}, c^{\prime}$ est le regard sur les signes et la communication qui domine : par exemple dans l'histoire du massacre de chats chez Darnton ${ }^{7}$, ou dans son tout récent livre Poésie et Police 8 , en Allemagne chez Kittsteiner ${ }^{9}$ (avec 
une intéressante référence théorique à Cassirer). Guilhaumou présente la Révolution française comme une révolution langagière et culturelle ${ }^{10}$, tandis que Chartier, lui, met en garde contre la tentation de fondre l'histoire dans l'élément langagier : la sémiose et la pratique - pour parler comme Vico: Homère et Hercule - sont pour lui des sphères qui ne peuvent s'interpénétrer intégralement ${ }^{11}$.
Foucault a inventé cet objet historique très particulier au-delà de l'homme agissant qu'il appelle discours et qui est structuré comme un langage ${ }^{12}$. Geertz admet une sémioticité radicale des sociétés dans leur ensemble: tout est texte ${ }^{13}$. Quant au philosophe Fellmann, inspiré par Vico, Schopenhauer et Dilthey, il conçoit "l'histoire comme un texte ${ }^{14} »$.

\section{L'historio-graphe}

Même si l'histoire parle, donc si les événements sont eux-mêmes de nature langagière, si ce qui arrive est partiellement, principalement, voire exclusivement communicationnel, il n'en reste pas moins que les événements ne forment pas un texte cohérent. Il n'y a plus personne pour croire qu'une storia ideale eterna se trouve à leur fondement. C'est précisément ici que se situe le problème de la science historique moderne : c'est celui du langage de l'histoire au sens second. Et c'est ici que nous rencontrons le second linguistic turn, manifestement le plus douloureux et le plus controversé. Mais, si moderne qu'il soit, il ne pose à vrai dire aucune question nouvelle.

On le sait, le classique Discours devant l'Académie de Humboldt sur la tâche de l'historiographe (1821) traite déjà du problème de la représentation de l'incohérence du déroulement événementiel ${ }^{15}$ : comment l'historien saisit-il les événements qui s'offrent à lui comme une masse chaotique et dans lesquels il se trouve comme dans une nappe de brouillard? comment les représentet-il ? comme écrit-il l'histoire ? Le terme «historio-graphe» est ici vraiment à prendre dans son sens littéral. Il y va de l'écriture. "Le déroulement événementiel n'est que partiellement visible dans le monde sensible, le reste doit être ressenti, déduit, deviné » (Humboldt, p. 35). Ressentir, déduire, deviner et « ajouter » (p. 36), telle est selon Humboldt la tâche de l'historien. Il est donc tout à fait hors de doute pour lui qu'il s'agit là d'une activité littéraire créatrice. C'est non par hasard, mais en suivant une tradition ancienne - Clio était une muse -, que Humboldt établit un parallèle entre l'historien et le poète. Ils ont l'imagination en commun. Mais l'imagination n'est pas - contrairement à ce que nous en faisons le plus souvent aujourd'hui le lieu d'un délire sans consistance ni rapport au monde. Humboldt entend toujours l'imagination au sens kantien $d$ 'une synthèse entre sensibilité et entendement, donc entre expérience du monde et rationalité. La créativité de l'historien ne s'épuise pas dans une invention langagière séparée du monde (et elle n'est naturellement pas davantage l'activité de l'artiste, qui consiste encore principalement pour Humboldt, de façon tout aristotélicienne, dans la mimesis de 
la nature). Le poète crée quelque chose qui n'était pas encore là. L'historien, lui, «ajoute»: il se trouve confronté aux sources des événements, un "squelette de faits donnés [Begebenheiten]" (p. 36), ce qui signifie qu'il a devant lui une réalité qu'il lui faut pénétrer par la pensée, une "matière » à laquelle il lui faut donner forme. Il se distingue donc du poète par son "sens du réel ». Cette forme particulière d'imagination, à la différence de sa forme poétique, présente un tropisme pour le monde. Avec le "sens du réel", on aborde le noyau de la théorie du langage : le rapport au monde, la référentialité.

Pour Humboldt, dont on sait qu'il était un philosophe du langage d'une certaine importance ${ }^{16}$, pour ce penseur du langage donc, il ne fait aucun doute qu'il y a au-delà de la langue et du texte une réalité, que c'est en tant qu'êtres de parole que nous sommes dans le monde, que nous nous référons au monde et nous expliquons avec le monde commun par la parole. Cependant, pour Humboldt, la parole se réfère au monde avec une force différente selon les tâches qui sont les siennes et le genre de discours qu'elle représente. Et c'est dans cette mesure que se modifie aussi le rôle de la langue. Ajoutons que, pour Humboldt bien plus encore que pour Vico, la «langue » n'est pas seulement le mot dans sa matérialité, vox, mais aussi le sens lié au mot : conceptus. La parole quotidienne, la «langue des affaires» et l'utilisation terminologique de la langue dans les sciences exactes laissent la langue pour ainsi dire derrière elles, elles se consacrent totalement aux choses, aux affaires (on trouve les deux sens dans le latin res), elles se livrent pour ainsi dire entièrement aux choses et aux affaires.
La sonorité particulière et le sens particulier sédimenté dans les langues, leurs «visions du monde» respectives, ne jouent aucun rôle dans ce type de parole ; Humboldt va même jusqu'à dire que ce qui fait la particularité propre de la langue en est précisément "éliminé ${ }^{17}$ ". La langue devient indifférente, "arbitraire» $[\ldots]$.

En revanche, et malgré son «sens du réel ", l'historiographie relève pour Humboldt, au même titre que la philosophie et la littérature, de ce type de discours qui reste auprès de la langue. La langue de l'histoire est un "emploi oratoire de la langue » dans lequel l'orateur accueille "le plein effet de la matière particulière de la langue » et où - pour le dire autrement - la langue "soit affecte d'elle-même et par son essence même la représentation des objets, soit est employée intentionnellement à cet effet » (p. 29 suiv.), c'est-à-dire pour la représentation des objets. [...].

Ce que l'on entend principalement aujourd'hui par le linguistic turn de l'historiographie - la prise de conscience de sa dimension langagière ou de sa littérarité - s'observe donc déjà chez Humboldt. Par ses réflexions, il récuse une conception naïve de l'historiographie qui pense celle-ci capable de simplement fixer par écrit le cours des événements tel qu'il s'offre à nous, pour ainsi dire de simplement l'enregistrer, à partir d'une théorie du langage assez naïve pour lui permettre de croire qu'elle peut «éradiquer » également le sens de la langue et du texte.

Roland Barthes lui-même, à qui on attribue, un siècle et demi plus tard, la responsabilité du linguistic turn des sciences historiques, n'écrit pas autre chose. L'objet principal de son célèbre 
article de 1967 sur «Le discours de $l^{\prime}$ histoire ${ }^{18}$ » est en effet la critique de l'illusion des historiens qui prétendent sortir de la langue et de sa sémantique et - plus radicalement encore qu'Aristote - relient la vox directement à la res, ou, pour l'exprimer de façon plus moderne, qui rapportent le signifiant directement au référent (il pense manifestement à l'historiographie de l'école des Annales, qui se revendique radicalement factuelle et «scientifique»). Tout comme Saussure, Roland Barthes sait que les mots ne sont pas simplement des noms qui renvoient à des choses, mais que ce sont des entités indissolublement bifides, des sons qui traînent leur signification avec eux, des unités composées d'un signifiant* et d'un signifie** (il ignore manifestement que Humboldt et Vico le savaient bien avant Saussure, ou encore que, pour Herder, les idées collent aux mots). Barthes insiste sur le fait que les contenus intratextuel et intralinguistique ne peuvent être «éradiqués ». Dans cette mesure, l'analyse de Barthes ne fait rien d'autre qu'inscrire dans le livre d'or des historiographes modernes une vérité que la théorie du langage connaît depuis Vico, Herder, Humboldt et Saussure.

Il n'en reste pas moins que Barthes - jetant ainsi le bébé historique avec l'eau $\mathrm{du}$ bain - radicalise son analyse du discours historiographique en ce sens qu'il en vient à lui dénier toute extériorité. Le rapport à un référent ne serait qu'un leurre, le référent n'existerait pas en dehors du texte. Barthes écrit : «Ce discours est sans doute le seul où le référent soit visé comme extérieur au discours, sans qu'il soit jamais possible de l'atteindre hors de ce discours ${ }^{19}$.
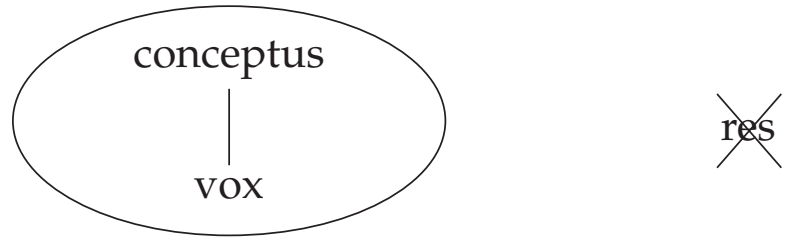

Pour Barthes en effet, les res gestae sont générées par le discours historique lui-même et n'existent pas en tant que telles en dehors de la parole. La distinction entre res gestae et historia rerum gestarum ne serait donc qu'un leurre. Ce que Humboldt nomme " l'élément ajouté » est ici mis en absolu. Le fait historique n'existe pour Barthes que dans la langue. Il n'y a pour ainsi dire rien à quoi on pourrait "ajouter " quelque chose. "Le fait n'a jamais qu'une existence linguistique ${ }^{* 20}$." Pourtant, tout se passerait dans le discours historique comme si le fait qui en soi n'existe que par le langage était la copie d'un élément extérieur à celui-ci, « comme si cette existence n'était que la copie pure et simple d'une autre existence, située dans un champ extra-structural, le "réel"*21 ». Ce qui est extérieur au langage, le réel, est pour Barthes un "comme si ", un leurre qu'il appelle « effet de réel* », l'effet de réel du discours historique. $\mathrm{D}^{\prime}$ après cette analyse, le sens du réel de Humboldt ne serait en quelque sorte qu'une chimère.

$$
\text { [...] }
$$

Une telle exclusion du monde ne peut naturellement que heurter les véritables historiens et leur « sens du réel »- lequel est à mon goût souvent par trop prononcé. Cependant, je n'ai encore jamais 
rencontré d'historien saisi par le linguistic turn qui serait prêt à défendre sérieusement l'immanence langagière radicale $\mathrm{du}$ discours historique. L'historien radicalement tourné vers le langage me semble être plutôt une cible en carton-pâte, un bouc émissaire, une sorte d'épouvantail (qui hante les travaux d'Iggers) chargé de transformer en repoussoir cette autre prise de conscience, passablement irrécusable celle-là, à savoir celle de la dimension langagière et de la littérarité incontournables du discours historique. Derrière la mise en avant $\mathrm{du}$ croque-mitaine se cache la nostalgie d'une scientificité "exacte", véritable, objective, telle qu'elle est censée exister dans les sciences « exactes », qui parlent une langue claire et exclusivement référentielle. C'est ainsi qu'Iggers invoque avec ferveur l'idéal linguistique de la philosophie analytique, l'idéal d'une langue claire et exclusivement référentielle, c'est-à-dire en fait l'aspiration à une absence de langage ${ }^{22}$.

En tout cas, il est un autre théoricien et historien à qui l'on prête bien à tort cette posture langagière excluant toute référentialité : je veux parler de Hayden White. Selon la lecture que j'en fais, White n'est en rien un propagandiste de la dimension langagière absolue $\mathrm{du}$ discours historique façon Roland Barthes, mais bien plutôt un critique de cette conception - du moins l'était-il à l'origine. Il fait la chose suivante : cette forme d'existence linguistico-littéraire $\mathrm{du}$ fait historique que Barthes n'avait fait que suggérer, il l'atteste effectivement chez différents historiens. Que Clio écrive en poétesse, pour reprendre le titre $d^{\prime}$ un de ses livres ${ }^{23}$, et de quelle façon elle écrit, Hayden White le constate dans ses nombreuses études métahistoriques. Mais au fond, il ne trouve pas cela bien du tout - du moins au début. Son premier grand livre était tendanciellement une critique de la littérarité de l'historiographie ; White (tout comme Iggers) y a plus d'une fois exigé la "scientificité", et donc l'objectivité de l'histoire sur le modèle des sciences exactes ${ }^{24}$. Certes, si je comprends bien ses derniers propos, il s'est fait entre-temps à l'idée que l'historiographie est littéraire - à des degrés divers, du reste -, c'est-à-dire qu'il a pris ses distances avec la volonté de la ramener sur le sentier de la désignation objective $^{25}$. Mais il ne va pas aussi loin que Barthes: il ne refuse pas aux historiens le "sens du réel ». Pas plus qu'aux poètes, d'ailleurs - et c'est à mes yeux sa faiblesse propre. Pour lui, la poésie aussi est référentielle, et c'est pour cela qu'il n'y a pas de différence entre les discours littéraire et historiographique. Donc: Melpomène, Polymnie et toutes les muses de la poésie s'expriment de façon référentielle. Mais c'est plutôt problématique $^{26}$. 


\section{Envoi}

Pour conclure, je voudrais illustrer de nouveau, à travers un exemple modeste, ce qui, dans la perspective de la théorie du langage, est le point décisif : la dimension langagière, ou la littérarité, du discours historique, qui n'exclut nullement sa référentialité. $L^{\prime}$ " événement vécu » auquel se réfère mon exemple est le signe que Willy Brandt envoie de Varsovie, un événement sémiotico-communicationnel que j'ai déjà eu l'occasion d'aborder. Voici ce que ma collègue Marie-Luise Recker écrit à propos de cet «événement vécu » dans son merveilleux petit livre qui vient de paraître sur l'histoire de la République fédérale :

Le symbole du nouveau commencement et de la réconciliation entre les deux États [la Pologne et l'Allemagne, J. T.] fut moins la signature du traité lui-même le 7 décembre 1970 que l'agenouillement du chancelier Brandt le même jour à l'occasion du dépôt de gerbe devant le monument des victimes du ghetto de Varsovie ${ }^{27}$.

Recker inscrit donc cet événement dans le récit de l'évolution de l'Ostpolitik et des rapports germano-polonais. J'ai moi-même - s'il m'est permis de me faire ici historien aux fins d'illustration - dit plus haut ceci du même événement :

Et quand les mots lui manquent, l'histoire paisible donne des signes tels que celui-ci, qui est sûrement le mot le plus émouvant et le plus signifiant de notre histoire contemporaine. Ce qui était à dire, l'Allemagne n'a pu le dire autrement qu'à travers ce geste.
J'ai pour ma part replacé cet événement dans une perspective plus longue et thématiquement plus large, pour ainsi dire dans l'histoire globale de l'Allemagne et avant tout dans l'histoire de la culpabilité de l'Allemagne concernant la guerre et l'Holocauste. Les deux propos contiennent, de façon massive, des «éléments ajoutés ", on ne peut pas dire que ce sont de simples constatations de ce qui s'est produit, du type : « Le 7 décembre 1970, un Allemand du nom de Willy Brandt s'agenouille devant le monument du ghetto ». Pourtant, ces deux extraits semblent être deux représentations possibles - et justes - de cet événement. Ils montrent chacun à quel point la mise en forme d'un sujet peut être différente. C'est la prise de conscience légitime du linguistic turn.

Cela ne signifie naturellement pas que la corporation des historiens ne puisse pas poser une série d'exigences autres concernant la forme nécessaire de tels propos afin qu'ils soient acceptés comme "scientifiques " et satisfassent aux règles du discours de leur discipline ${ }^{28}$. Mais que l'on puisse dire des choses différentes et des vérités différentes sur le même sujet ne devrait pas remettre sérieusement en question le critère de scientificité. On pourrait même admettre avec Vico que seul un regard multiple sur le sujet constitue la «scientificité » de l'histoire.

Mais, avant tout, nos descriptions et interprétations différentes et générées par le langage ne sont pas une raison pour douter de la factualité de ce qui se produit, ni pour renoncer à ce que Chartier appelle l'archive ${ }^{29}$ : il y a suffisamment de preuves pour savoir que, le 7 décembre 1970, un individu précis, 
investi à Varsovie d'une fonction politique précise, s'est effectivement agenouillé en ce lieu très précis, et qu'il s'agissait là d'un acte sémiotique intentionnel. Brandt n'a pas simplement fait une chute, par exemple parce qu'il aurait perdu connaissance. De fait, son geste a été compris de tous comme un signe. Cet acte sémiotique lui a même valu le prix Nobel : le prix Nobel de la paix pour un mot de l'histoire. Il ne fait donc aucun doute pour la plupart des participants au jeu de langage de l'histoire que nos phrases se réfèrent à des res gestae, mais cela vaut pour tout participant à peu près normal au jeu de notre monde sociétal. Et il doit en être ainsi, sans quoi le mondo civile s'effondrerait, pris de vertige entre fiction et réalité (ce qu'il fait déjà passablement, mais nous avons encore la plupart du temps conscience que cela n'est pas dans l'ordre des choses). Si le linguistic turn remettait vraiment sérieusement en question la réalité extérieure au texte, au langage, alors il n'aurait pas seulement fait prendre conscience de la dimension langagière de l'historiographie déjà reconnue par Humboldt, mais représenterait aussi une forme de folie : la perte de la réalité.
C'est sur cette constatation plus psychiatrique que linguistique que j'achèverai mes considérations sur la langue de l'histoire-la lingua in cui parla la storia (l'éternelle tout comme la nôtre) -, terme de mon petit essai concernant la linguistique de l'histoire. Et je conclus sur une remarque qui fait également éclater le cadre de ma discipline et aurait plutôt sa place dans le conseil en développement personnel, voire dans la direction de conscience. Mais je la fais tout de même, et je la fais avec une pointe de jalousie, car la chance sourira toujours plus aux historiens qu'à nous autres linguistes. Il s'agit encore une fois du prix Nobel : il y a cent ans exactement, en 1902, Theodor Mommsen ${ }^{30}$ recevait le prix Nobel, mais le prix Nobel de littérature, pas de science historique. Le prix Nobel pour la langue de l'histoire. Si ce fait n'a pas seulement une existence linguistique, si donc il est réellement arrivé - cela fait un petit bout de temps, je sais, mais l'archive dit qu'il en a bien été ainsi -, alors la corporation des historiens devrait ne pas avoir peur du linguistic turn, c'est-à-dire de la littérature ${ }^{31}$, mais puiser dans ce fait historique fierté et encouragement à une belle écriture de l'histoire.

Traduit de l'allemand par Philippe Forget

\section{Notes}

* En français dans le texte [NDT].

1 Référence à Cicéron: «Quand les armes parlent, les lois se taisent » [NDT].

2 Ulrich BECK : Das Schweigen der Wörter. Über Terror und Krieg, Francfort-sur-le-Main, Suhrkamp, 2002.

3 Ce terme (en français: tournant linguistique), qui revient à de nombreuses reprises dans le

texte de Jürgen Trabant, renvoie au philosophie américain Richard Rorty, pour qui la réalité ne peut être pensée en dehors de la médiation du langage. Dans sa version extrême, la réalité demeure radicalement hors langage, et le langage seul constitue alors une réalité tangible [NDT].

4 Roger Chartier, Die unvollendete Vergangenheit. Geschichte und die Macht der Weltauslegung, 
Berlin, K. Wagenbach, 1989 ; ID., «L'histoire culturelle entre "lingustic turn" et retour au sujet », dans Hartmut LeHMANN (dir.): Wege zu einer neuen Kulturgeschichte, Göttingen, Wallstein, 1995, p. 29-58.

5 Robert Darnton, Le Grand Massacre des chats. Attitudes et croyances dans l'ancienne France, traduit de l'anglais (États-Unis) par Marie-Alyx Revellat, R. Laffont 1985 ; repris en 2011 aux Belles-Lettres [NDT].

6 Natalie Zemon Davis, Pour sauver sa vie. Les récits de pardon au XVI siècle, traduit de l'anglais (ÉtatsUnis) par Christian Cler, Seuil, 1988 [NDT].

7 Robert DARnton, op. cit. Darnton s'intéresse ici, à travers des témoignages d'inconnus dénués de toute prétention littéraire, aux mentalités d'une époque et à leur représentation. Le titre $\mathrm{du}$ livre reprend le sujet du deuxième chapitre («Une révolte d'ouvriers: le grand massacre des chats de la rue Saint-Séverin »), étude de cas dans laquelle des domestiques tuent symboliquement leurs maîtres à travers l'assassinat $\mathrm{d}$ 'une chatte qui leur était chère [NDT].

8 ID., L'Affaire des Quatorze. Poésie, police et réseaux de communication à Paris au XVIII siècle, traduit de l'anglais (États-Unis) par Jean-François Sené, Gallimard, 2014 [NDT].

9 Heinz Dieter Kittsteiner, «Was heißt und $\mathrm{zu}$ welchem Ende studiert man Kulturgeschichte? », Geschichte und Gesellschaft, vol. 23, no 1, 1997, p. 5-27 [NDT].

10 Jacques Guilhaumou, La Langue politique et la Révolution française. De l'événement à la raison linguistique, Méridiens Klincksieck, 1989 [NDT].

11 Roger Chartier, art. cit.

12 Michel Foucault, Les Mots et les Choses. Une archéologie des sciences humaines, Gallimard, 1966.

13 Clifford Geertz, The Interpretation of Cultures, New York, Basic Books, 1973.

14 Ferdinand Fellmann, "Geschichte als Text. Ein Plädoyer für die Geschichtsphilosophie », Information Philosophie, $\mathrm{n}^{\circ}$ 4, 1991, p. 5-14.

15 Wilhelm von Humboldt : « Ueber die Aufgabe des Geschichtsschreibers », dans Wilhelm von Humboldts gesammelte Schriften, édit. Albert Leitzmann, Berlin, Behr, 1905, vol. 4, 1820-1822, p. 35-56; repris et commenté dans Wilhelm von Humbold, Über die Sprache. Reden vor der Akademie, édit. Jürgen Trabant, Tübingen/Bâle, Francke, 1994.

16 Voir Jürgen Trabant, Apeliotes oder Der Sinn der Sprache. Wilhelm von Humboldts Sprach-Bild, Munich, Fink, 1986 ; et Traditionen Humboldts, Francfort-sur-le-Main, Suhrkamp, 1990. En français: Humboldt ou le Sens du langage, édition française élaborée par l'auteur et François Mortier avec la collaboration de Jean-Luc Evard, Liège, Mardaga, 1992 ; et Traditions de Humboldt, traduit de l'allemand par Marianne Rocher-Jacquin, préface d'Henri Meschonnic, Éd. de la Maison des sciences de l'homme, 1999.

17 Wilhelm von Humboldt: «Ueber das vergleichende Sprachstudium in Beziehung auf die verschiedenen Epochen der Sprachentwicklung » [1820], dans Wilhelm von Humboldts gesammelte Schriften, op. cit., p. 1-34, ici p. 30 ; repris et commenté dans Wilhelm von Humbold, Über die Sprache. Reden vor der Akademie, op. cit.

18 Roland BARthes, «Le discours de l'histoire » [1967], dans OEuvres complètes, t. 2, 1966-1973, édit. Éric Marty, Seuil, 1994, p. 417-427.

19 Ibid., p. 425.

20 Ibid.

21 Ibid.

22 Georg G. IGGERS, Geschichtswissenschaft im 20. Jahrhundert, 2e éd., Göttingen, Vandenhoeck \& Ruprecht, 1996, p. 89.

23 Il s'agit ici en réalité du titre de la traduction allemande de Tropics of Discourse. Essays in Cultural Criticism, Baltimore, Johns Hopkins University Press, 1978. Cet ouvrage est en effet paru en Allemagne sous le titre: Auch Klio dichtet oder die Fiktion des Faktischen. Studien zur Tropologie des historischen Diskurses, Stuttgart, Cotta-Klett, 1986 [NDT].

24 Hayden White, Metahistory. The Historical Imagination in Nineteenth-Century Europe, Baltimore, Johns Hopkins University Press, 1973, XI, 2, p. 428.

$25 I D$, «Entgegnung auf Georg G. Iggers », Geschichte und Gesellschaft, vol. 27, $\mathrm{n}^{\circ} 2$ 2, 2001, p. 341-349. 
$26 C^{\prime}$ est pour le moins le problème central de l'esthétique littéraire, que l'on ne peut en rien considérer comme résolu. À ce propos, voir les points de vue divergents par exemple chez Joachim KüPPER, "Was ist Literatur? », Zeitschrift für Ästhetik und allgemeine Kunstwissenschaft, vol. 45, $\mathrm{n}^{\circ} 2$, 2001, p. 187-215, et Jürgen Trabant, Elemente der Semiotik, $3^{\mathrm{e}}$ éd. Tübingen, Francke, 1994, chap. 10 et 11.

27 Marie-Luise RecKer, Geschichte der Bundesrepublik Deutschland, Munich, Beck, 2002, p. 72 suiv.

28 Voir Roger Chartier, art. cit., p. 55.

29 Ibid.

30 L'historien allemand Theodor Mommsen (1817-1903) est considéré comme l'un des plus importants spécialistes de l'Antiquité, et plus précisément de l'Antiquité romaine. C'est du reste son Histoire romaine [Römische Geschichte], rédigée au début de sa carrière (trois volumes parus entre 1854 et 1856), qui lui vaut l'attribution du prix Nobel de littérature en 1902. Il a également été fait citoyen d'honneur de la ville de Rome [NDT].

31 Voir Peter ScнӧтtLeR, « Wer hat Angst vor dem "linguistic turn"? », Geschichte und Gesellschaft, vol. 23, no 1, 1997, p. 134-151. C'est dans ce sens que Christian Meier - par-delà l'agitation causée par le linguistic turn - plaide aussi pour une "nouvelle historiographie des historiens » satisfaisant à des exigences de qualité littéraire. Voir Christian MeIER, « Programm einer Geschichtsschreibung ", Merkur, n ${ }^{\circ}$ 528, 1993, p. 207-217, ici p. 208. 